\title{
La política de habitualidad y su efecto en la regulación de las operaciones con partes relacionadas
}

\author{
Gonzalo Islas Rojas* \\ Osvaldo Lagos Villarreal**
}

\begin{abstract}
RESUMEN
Este trabajo analiza cómo la regulación de las operaciones habituales afecta la eficacia de la normativa respecto de operaciones entre partes relacionadas, para ello se analizan dogmáticamente los fundamentos y problemas de la regulación de operaciones habituales y se revisa empíricamente cómo se autorregulan las sociedades del IPSA, mediante la comparación de aspectos de procedimiento y sustantivos contenidos en las políticas de habitualidad producidas por las propias sociedades. Se observa que las sociedades revisadas son reacias a autorregularse, lo que incrementa el riesgo de tunneling $y$, además, se concluye que existe un amplio espacio para mejoras en la regulación, sin volverla excesivamente gravosa.
\end{abstract}

Operaciones entre partes relacionadas - operaciones habituales - gobiernos corporativos

\section{Ordinary course of business policy and its effect on related party transactions regulation}

\begin{abstract}
This paper analizes how ordinary course of business transactions regulation affects the effectivity of the related party transactions law, for which the foundations and problems of ordinary course of business transactions are doctrinally analized, and self-regulation of IPSA corporations are empirically checked, by the comparison of procedimental and substantive aspects contained in ordinary
\end{abstract}

* Licenciado en Economía, Universidad de Chile. Doctor en Economía, Universidad de California, Los Angeles, Estados Unidos. Profesor de Economía, Universidad Adolfo Ibáñez. Correo electrónico: gonzalo. islas@uai.cl.

** Licenciado en Ciencias Jurídicas y Sociales, Universidad Adolfo Ibáñez. Doctor en Derecho, Universidad de los Andes. Profesor de Derecho Comercial, Universidad Adolfo Ibáñez. Correo Electrónico: osvaldo.lagos@uai.cl.

Queremos agradecer los comentarios de los participantes en el seminario interno de profesores de la Facultad de Derecho de la Universidad Adolfo Ibáñez y los comentarios de dos árbitros anónimos. En particular, agradecemos la dedicada y eficiente ayuda de Iván Cerda en la elaboración de este trabajo.

Artículo recibido el 30 de abril de 2018 y aceptado para su publicación en este número el 18 de enero de 2019. 
transactions policies produced by those corporations. It is observed that chilean corporations are reluctant to self-regulate, which increments the risk of tunneling and, also, it is concluded that there is ample scope for improvements in regulation, without turning it excessively burdensome.

Related party transactions - ordinary course of business transactions - corporate governance

\section{INTRODUCCIÓN}

$\mathrm{L}$ a Ley $\mathrm{N}^{\circ} 20.382$ de 2009, que introdujo, entre otras reformas pertinentes a gobiernos corporativos, una nueva regulación acerca de operaciones con partes relacionadas (en adelante, OPR), permitió que las propias sociedades anónimas determinaran qué transacciones deberían quedar exentas del procedimiento de aprobación contemplado en la primera parte del artículo 147 de la Ley N 18.046 de 1981 (en adelante, LSA), por ser de realización ordinaria. No obstante, al hacerlo no definió parámetros con los que se debía determinar qué operaciones serían consideradas habituales, con ello, la definición de la política de habitualidad quedó entregada al criterio de los propios regulados.

Ante esto, cabría esperar que las reacciones de los regulados oscilaran entre dos extremos: el primero, diseñar una política que determine mecanismos internos de aprobación y fiscalización de estas operaciones y de aseguramiento para que la OPR cumpla con las exigencias sustantivas (interés social y condiciones de mercado) y que precise cuáles operaciones son aquellas que se pueden considerar habituales, con la finalidad de dar fluidez a la administración de la sociedad, pero disminuyendo el riesgo de tunneling ${ }^{1}$; la segunda, diseñar una política lo más amplia y ambigua posible, con la finalidad de evitar la aplicación de las reglas respecto de OPR y la supervisión del fiscalizador, facilitando la administración de la sociedad y, al mismo tiempo, facilitando la realización de tunneling ${ }^{2}$.

${ }^{1}$ JoHnson et al., 2000, pp. 22-27 en su trabajo titulado Tunneling, introdujeron esta expresión en la literatura de gobiernos corporativos, que designa genéricamente las formas cuando los controladores de sociedades pueden apropiarse de activos que corresponden a toda una sociedad anónima y, por tanto, a todos los accionistas. Así, se puede hacer tunneling en perjuicio de los accionistas en general, o en perjuicio de los accionistas minoritarios, pues lo anterior depende de la estructura de control.

2 Explica PAz-Ares, 2004, pp. 10-15, que es usual que las mejoras a los gobiernos corporativos sean vistas con reticencia por las sociedades cotizadas en un primer momento, hasta que se toma conciencia de su impacto positivo en el valor de la empresa. Por su parte, Ross, 2013, pp. 47-53, señala que ciertas complementariedades y factores estructurales determinan que, lejos de haber incentivos para modificar las estructuras grupales concentradas, características de Latinoamérica, exista una tendencia reforzada a mantener el statu quo institucional. Por todo esto, es posible esperar resistencia ante regulación que implique mayores niveles de transparencia y competitividad. En consecuencia, con mayor razón es esperable que las sociedades anónimas abiertas sean reacias a la autorregulación, como se observó en la reacción ante los requerimientos informativos de la Norma de Carácter General No 341, según informó en su oportunidad la Superintendencia de Valores y Seguros (en adelante, CMF), actual Comisión para el Mercado Financiero (en adelante, CMF). CMF, 2015, p. 6: "De las autoevaluaciones remitidas, se pudo observar que las entidades en su mayoría 
La cuestión es relevante, pues mediante la política de habitualidad se puede ampliar o restringir, en los hechos, el ámbito de aplicación de las reglas acerca de OPR, con lo que la política de habitualidad se erige como un factor relevante para la eficacia de la regulación de estas operaciones y para la protección de inversionistas.

La literatura especializada reconoce que las OPR pueden ser utilizadas como un mecanismo que permite la extracción de valor por parte de los controladores, en desmedro de los inversionistas minoritarios (hipótesis de agencia). Al mismo tiempo, se reconoce que las OPR pueden agregar valor a la empresa, ya que reducen los costos de transacción y eliminan problemas de oportunismo postcontractual (hipótesis de eficiencia) ${ }^{3}$. Debido a lo anterior, las políticas de habitualidad son un mecanismo que, en teoría, permite separar aquellas transacciones propias del funcionamiento habitual de la empresa de aquellas que pueden tener fines expropiatorios.

En este trabajo se observan las políticas de habitualidad de las sociedades anónimas que integran el Índice de Precio Selectivo de Acciones de la Bolsa de Comercio de Santiago (IPSA), con la finalidad de conocer cuál ha sido, en términos generales, la reacción de las sociedades anónimas más relevantes del mercado chileno ante la oportunidad de autorregularse en esta materia. Esta observación permite evaluar el efecto de la política de habitualidad en la eficacia de la regulación entre OPR, al menos desde una perspectiva del diseño institucional. De este modo, se analiza la forma cuando se construye normativamente la regulación y su efecto previsible en la consecución de sus objetivos, para contribuir a la adecuada comprensión del funcionamiento del mercado ${ }^{4}$.

tendieron a dar un cumplimiento formal a la normativa, centrándose en responder de manera acotada las respuestas, sin entregar mayores antecedentes que facilitaran la comprensión de las prácticas adoptadas por la entidad ni tampoco respecto de la adopción de prácticas distintas a las contenidas en la normativa. A su vez, se observó que no hubo cambios significativos en las prácticas adoptadas ni a enriquecer las respuestas provistas la primera vez, lo que tiende a reafirmar la ausencia de interés manifiesto de la entidad por mejorar sus prácticas o dar a conocerlas de mejor manera al público" (cfr. CENTro DE Gobierno Corporativo UC, 2015 , p. 3, que coincide con el análisis del regulador pero aclara que la reticencia se debe a que las políticas sugeridas en la norma no son consideradas buenas prácticas por los emisores regulados, lo que, en nuestra opinión, no hace más que confirmar las tendencias explicadas por PAZ-Ares y Ross.

${ }^{3}$ ENRIQUes, 2014, p. 2-9, discute los principales fines que pueden tener las OPR y destaca que ellas pueden ser especialmente importantes desde la perspectiva de eficiencia en economías donde las instituciones sean débiles y, por tanto, el cumplimiento de los contratos menor. Dada esta dicotomía entre los fines que pueden tener las OPR, no resulta sorpresivo que la evidencia empírica entregue resultados mixtos en relación con el efecto de las OPR sobre el valor de la empresa. Véase BONA SÁNCHEZ et al., 2017, pp. 5-6 para una discusión reciente sobre los principales resultados de los estudios empíricos.

${ }^{4}$ Esto es, una perspectiva de institucionalismo legal (DEAKIN et al., 2017, pp. 188-200). 


\section{El SENTIDO DE LA POLÍtica GENERAL DE HABITUALIDAD Y LOS RIESGOS QUE ENCIERRA}

\section{1. ¿Qué es una política de habitualidad?}

La política de habitualidad es un acuerdo del directorio de una sociedad anónima abierta, conforme a ello se determinan las condiciones para que ciertas transacciones, que quedan comprendidas en la definición de operación con partes relacionadas del artículo 146 LSA, no deban someterse al procedimiento para su aprobación contemplado en el artículo 147 LSA (denominado "procedimiento reglado" por los autores de la ley), por ser de celebración ordinaria entre la sociedad de referencia y su contraparte relacionada.

El hecho de que una operación sea "habitual", en los términos indicados en el inciso anterior, se erige como una de las tres excepciones a la aplicación del procedimiento para la aprobación de $\mathrm{OPR}^{5}$. La forma de hacer efectivas estas excepciones está regulada en el Nuevo Reglamento de Sociedades Anónimas, Decreto Supremo de Hacienda N 702 de 2011 (en adelante, NRSA). El NRSA señala que para hacer efectiva la excepción al procedimiento reglado, es necesario que el directorio "adopte de forma expresa una autorización de aplicación general” (artículo 171 NRSA). La intención de los redactores del reglamento parece haber sido que la política general de habitualidad de cada sociedad anónima abierta se insertase en la "autorización de aplicación general", dándole la debida publicidad a los criterios para la aplicación de las respectivas excepciones al procedimiento de aprobación de $\mathrm{OPR}^{6}$. No obstante, lo que se observa en la mayoría de los casos es solo la información y publicación independiente de la política general de habitualidad, en conformidad con lo dispuesto en la letra b del artículo 147 LSA.

\section{2. ¿Por qué es necesaria una politica general de habitualidad?}

En el ordenamiento jurídico chileno, la estrategia para la regulación de OPR considera la imposición de exigencias sustantivas o estándares, reglas referidos a información y reglas de procedimiento para la adopción del acuerdo social con el que se celebrará el contrato entre partes relacionadas ${ }^{7}$. Las primeras exigen, en conformidad con las reglas generales, que las operaciones se realicen en condiciones de mercado y con la finalidad de promover el interés social (artículo 147 inciso primero LSA). Las segundas, tienen por finalidad conceder a quienes representan a los intereses protegidos por la regulación, o a los propios accionistas, la oportunidad de conocer la celebración de la operación con partes relacionadas para, de este modo, adoptar medidas para la protección de estos intereses

${ }^{5}$ Los otros dos consisten en que la operación no sea de monto relevante (artículo 147 inciso final letra a LSA) y en que la sociedad de referencia posea al menos $95 \%$ de la propiedad de la contraparte relacionada (artículo 147 inciso final letra c LSA).

${ }^{6}$ Eyzaguirre y Valenzuela, 2015, p. 284.

${ }^{7}$ Acerca de las múltiples alternativas para la regulación de este tipo de operaciones, véase ENRIQUES (2014) pp. 14-27. 
(artículo $147 \mathrm{~N}^{\mathrm{os}}$ 2, 3 y 6, artículo 50 bis $\mathrm{N}^{\circ}$ 3). Las terceras determinan una forma para la adopción de la decisión, con una triple finalidad: permitir el flujo de información previa, relativa a la operación, tanto a los accionistas como al mercado; excluir de la adopción de la decisión a las partes interesadas, aunque solo parcialmente en el caso chileno ${ }^{8}$; facilitar la aplicación de las reglas concernientes a responsabilidad de directores 9 .

Las reglas de procedimiento para la adopción de acuerdos que permitan la celebración de OPR, presentan ciertas ventajas para evitar tunneling. El solo hecho de que se discuta si una operación propuesta es o no operación entre partes relacionadas, hace reaccionar al mercado, que en caso de sospechar tunneling, se manifiesta haciendo bajar el precio de la sociedad de referencia, lo que determina un mecanismo de presión bastante efectivo, aunque con efectos dañinos colaterales ${ }^{10}$. Además, la información que se provee al mercado a propósito del procedimiento para la adopción de OPR, en particular los informes de evaluadores independientes, establece las bases para llegar a un acuerdo con los accionistas no implicados en la operación, lo que, a su vez, permite destrabar la realización de la operación y acordar condiciones que, usualmente, resultan ser más equitativas para todos los accionistas, aunque no necesariamente justas ${ }^{11}$.

Más allá de los evidentes defectos de este procedimiento ${ }^{12}$, resulta ser, como se ha sostenido, un mecanismo eficaz para mejorar la posición de minoritarios en grandes

${ }^{8}$ Esto solo ocurre a propósito de la decisión de los directores, pero no respecto de la votación de la junta de accionistas.

${ }^{9}$ Si adoptan la decisión de llevar a cabo la operación con partes relacionadas, es muy difícil que puedan incurrir en responsabilidad civil si es que la operación ha respetado cada una de las exigencias del procedimiento, a pesar de que pudiese sospecharse que no persigue el interés social o que no se ha celebrado en condiciones de mercado. En cambio, a pesar de que pueda considerarse que la operación cumple con las condiciones sustanciales, si no se observa el procedimiento legal, serán lo demandados quienes deban demostrar que la operación satisface las exigencias sustantivas del artículo 147 LSA (en el mismo sentido Barros, 2006, p. 847, Atria, 2015, pp. 23-24, Eyzaguirre y Valenzuela, 2015, p. 293).

${ }^{10}$ Así ocurrió, por ejemplo, en el caso del aumento de capital de Enersis en 2012-2013. El problema de este mecanismo es que, a causa de la poca visibilidad del enforcement de las reglas de OPR, los inversionistas castigan no solo a la sociedad a la que afecta un caso, sino que a todas las sociedades pertenecientes al mismo mercado, pues todas ellas están sujetas al mismo marco legal, que el caso específico revela como vulnerable a la realización de tunneling.

${ }^{11}$ Por consiguiente, se observa que si bien las condiciones de la operación han mejorado para minoritarios a partir de este mecanismo, la operación se realiza de todas formas, aun cuando no tenga por finalidad promover el interés social, sino que el interés de la matriz del grupo. Al final, una solución negociada suele ser más favorable para la parte con mayor poder de negociación (véase FISs, 1984, pp. 1073-1089), por lo que un mecanismo que conduce usualmente a una solución negociada no es necesariamente el mejor mecanismo para los minoritarios. En términos de su diseño, el procedimiento para la adopción de OPR presenta varios puntos débiles: primero, se cuestiona la propia efectividad de los informes de evaluadores independientes como mecanismo de control, a partir del problema de independencia de los evaluadores (que obtienen más beneficios por otros roles de la banca de inversión que de la elaboración de informes respecto de OPR, como sostiene ENRIQUEs, 2014, p. 25; segundo, la ausencia de una regla de mayoría de la minoría para la adopción de la decisión en la junta de accionistas, lo que facilita la adopción de la decisión de parte del controlador (Kershaw, 2012, pp. 704-705); tercero, el hecho de que la infracción a las reglas del artículo 147 LSA no suponga la invalidación o impugnabilidad de la decisión, sino solo indemnización de perjuicios y el reembolso de los beneficios.

${ }^{12}$ En particular, la ausencia de una regla de mayoría de la minoría en la eventual votación de la junta extraordinaria de accionistas para la aprobación de la operación. Lo anterior significa que no se cumpla con 
operaciones, aunque no necesariamente para evitar operaciones de tunneling ${ }^{13}$. Sin embargo, el procedimiento es bastante costoso, cuestión que, en cierto modo, lo vuelve impracticable para operaciones de bajo monto o usuales de una sociedad, donde el remedio se hace peor que la enfermedad. Por lo anterior, en todas las jurisdicciones donde se incorpora una regulación de las $\mathrm{OPR}$, se reconocen reglas de excepción para este tipo de operaciones.

Como se revisa en el próximo apartado, en algunos casos estas excepciones están determinadas por el monto de las operaciones y, en otros, ellas están definidas por su calificación por la propia sociedad como operaciones habituales, o bien se combinan ambos factores. La regulación chilena se inserta en este tercer grupo, esto es, las excepciones están determinadas por ambos factores, además de una regla de concentración de la propiedad de la matriz de referencia sobre la filial con la que opera, que se explica por el hecho de que los colegisladores han estimado que, en este caso, el conflicto de intereses no es suficientemente relevante ${ }^{14}$.

\section{La habitualidad en otras jurisdicciones}

Como es lógico, todos los ordenamientos que reconocen una regulación en las OPR, deben contemplar, por las razones expresadas en el apartado anterior, una regla para lidiar con el problema de las transacciones ordinarias o de bajo monto. A continuación se examinan brevemente tres regulaciones europeas, pues ellas utilizan o han utilizado criterios similares a los establecidos legalmente en Chile para resolver esta cuestión. El sentido de este apartado es verificar si el problema de la habitualidad es un inconveniente común entre aquellas jurisdicciones que deciden regular el obstáculo del potencial tunneling entre sociedades relacionadas por medio de la técnica legislativa OPR, o bien,

\footnotetext{
las mínimas exigencias de una regulación acerca de conflictos de intereses, pues se admite que el accionista interesado pueda votar para la aprobación de la operación en la que puede realizar tunneling. En cambio, la regla sí es reconocida en Gran Bretaña, y es considerada una regla crucial, pues no solo evita operaciones que no sean celebradas a precio de mercado, sino incluso porque "desincentiva a cualquier inversionista para convertirse en accionista controlador por el solo hecho de creer que puede usar su poder como controlador para beneficiarse a sí mismo (Kershaw, 2012, p. 705).

${ }^{13}$ Si bien en los hechos se observa que las operaciones se acercan un poco más al cumplimiento de la compleja y difícilmente demostrable exigencia de realizar la operación según condiciones de mercado, no necesariamente se justifica que la operación haya sido realizada a favor del interés social. Por el contrario, puede subsistir la sospecha de que la operación es realizada en beneficio de los intereses de la matriz (cfr. LEFORT y Wigodski, 2007, pp. 92-96, a propósito de la venta de Telefónica Móvil de Chile S.A. por Telefónica CTC Chile a Telefónica Móviles S.A., estas últimas ambas filiales de Telefónica S.A.

${ }^{14}$ En efecto, las reglas sobre OPR en Chile protegen los intereses de los accionistas, sean todos los accionistas en el caso de que el relacionado es un director o ejecutivo, sean los minoritarios en el caso en que el relacionado es una sociedad vinculada con el controlador. Este último caso es el más relevante en Chile. Cuando se trata de este último caso, el problema se atenúa si la sociedad de referencia es titular del 95\% o más de las acciones de la filial, pues el beneficio que puede obtener un accionista de la filial por una operación que no sea realizada a precio de mercado es escaso. No debe confundirse lo anterior con el caso inverso, pues el beneficio que puede obtener la matriz en perjuicio de la filial puede ser cuantioso, como demuestra el caso Pehuenche.
} 
si, por el contrario, existen soluciones claras a este respecto en otras jurisdicciones y, en consecuencia, el problema de la habitualidad es solamente una complicación de la regulación chilena cuya solución se puede lograr fácilmente por medio de la incorporación de una regla adecuada.

En Francia, las OPR se declaran en conformidad con la norma contable IAS 24 y a la regulación de las convention réglamentées ("acuerdos regulados”), que constituyen el equivalente funcional a las OPR ${ }^{15}$. Los “acuerdos regulados" declaran como "acuerdo libre", es decir, no sujeto a las reglas especiales de aprobación de este tipo de transacciones, a las "operaciones corrientes que se celebran en condiciones normales" (Código de Comercio francés, art. L. 225-39). La jurisprudencia francesa ha declarado que lo que determina que la operación sea corriente no es un estándar objetivo de la profesión, sino que los propios usos de cada sociedad ${ }^{16}$. Desde luego, el hecho de que la determinación de que un cierto acuerdo es un "acuerdo libre" corresponda a la propia sociedad controlada, ha sido criticado por parte de la doctrina ${ }^{17}$. No obstante, el estado de la cuestión en Francia ha evolucionado en un sentido desfavorable a los accionistas minoritarios. Hasta antes del 2014 el artículo R 225-32 del Código de Comercio francés obligaba al Presidente del Consejo de Administración a informar al Consejo de Administración y a los Comisarios de Cuentas (órganos equivalentes al directorio y a los auditores externos) respecto de la lista y el objeto de las convenciones declaradas como convenciones libres. No obstante, el artículo R 225-32 del Código de Comercio francés fue derogado ${ }^{18}$. En opinión de una firma de proxy advisors francesa, la reforma de 2014 "ha más bien destruido el control de OPR en una forma muy desapercibida", generando "un grave retroceso en el control de los accionistas sobre las operaciones con conflictos de intereses"19.

En el caso de Italia, el regulador (Commissione Nazionale per le Societá e la Borsa, o Consob) dictó en 2010 una completa regulación de las OPR para sociedades cotizadas. La regulación es diferenciada dependiendo de la magnitud de las operaciones y, además, algunas de sus reglas pueden ser modificadas (haciéndolas más o menos exigentes) por los códigos de gobierno corporativo interno de las respectivas sociedades. En este sentido, las sociedades reguladas pueden excluir tanto del procedimiento de aprobación como de

15 OECD, 2012, p. 63.

16 Germain y Magnier, 2009, p. 458.

17 En este sentido, SснміDт, 2009, pp. 12-13: "se puede comprender que las transacciones no significativas que sean celebradas en condiciones normales no figuren en la lista prevista por los textos citados, pues ellas no suponen, prima facie, un riesgo de anomalía significativa en la contabilidad. Pero si se revisa la cuestión no desde el punto de vista de la regularidad y sinceridad de la contabilidad, sino desde el punto de vista de la calidad del gobierno corporativo de la sociedad, la omisión de estas operaciones de la lista de transacciones entre partes relacionadas produce el efecto de sustraerlas del conocimiento de los accionistas y de control. El control es necesario para asegurar que las transacciones entre partes relacionadas son celebradas conforme a condiciones normales y no presentan una relevancia significativa: no es natural dejar esta verificación en poder de los propios contratantes; esto elimina el poder del juez, que no puede de este modo conocer la existencia misma de las operaciones que no le son reveladas".

${ }^{18}$ Por el decreto 2014-1063 de 18 de septiembre de 2014.

${ }^{19}$ Proxinvest, 2014, p. 2. 
la obligación de información, a aquellas transacciones celebradas en el curso ordinario del negocio y en términos equivalentes a aquellos que prevalecen en transacciones sin conflictos de intereses. Asimismo, se pueden excluir aquellas transacciones que, estando bajo el límite para que la operación sea considerada relevante, queden comprendidas dentro del límite de operaciones de bajo monto. La determinación concreta de ambas excepciones, esto es, de lo que constituye una operación de bajo monto y de lo que constituye una operación celebrada en el curso ordinario del negocio, corresponden a la respectiva sociedad. No obstante, si una operación exceptuada del control por considerarse propia del curso ordinario del negocio, sobrepasa el monto para ser considerada operación relevante, debe ser informada al regulador ${ }^{20}$.

En el mismo sentido, en Gran Bretaña la Listing Rule 11.1, que regula las OPR para sociedades listadas en premium listing, admite que el procedimiento de aprobación e información que contempla no se aplique al tratarse de una operación "celebrada en el curso ordinario de los negocios", ni tampoco al tratarse de operaciones de "bajo monto" (small transactions) $^{21}$. Si bien la propia sociedad determina si una operación queda comprendida en la excepción, el regulador (Financial Conduct Authority o FSA) puede revisar que no se trate de un uso abusivo de la excepción, aunque se ha sostenido que "no está claro cuán rigurosamente la FCA revisa operaciones calificadas por el emisor como del curso ordinario de los negocios" 22 . No obstante, cuando una sociedad va a celebrar una operación entre partes relacionadas, debe consultar a su sponsor, quien debe verificar que la excepción no sea aplicada abusivamente ${ }^{23}$.

De todas formas, tanto las sociedades francesas, italianas e inglesas están obligadas a reportar según reglas IFRS desde 2005, por lo que se les aplica la Norma Internacional de Contabilidad 24 a las OPR, lo que permite tener acceso a información respecto del importe de las transacciones y de sus saldos pendientes ${ }^{24}$. De este modo, quienes puedan revisar la contabilidad completa de la compañía (insiders y reguladores) pueden acceder a esta información. El problema es que, en muchas ocasiones, no son los accionistas minoritarios los que tienen acceso a la contabilidad completa. Además, aun si los minoritarios tienen acceso a la información, la operación ya ha sido celebrada y, por lo general, las tutelas a posteriori, como la responsabilidad civil o la restitución de beneficios, son poco

${ }^{20}$ BIANCHI et al., 2014, p. 13.

${ }^{21}$ Estableciendo cuatro criterios o ratios a partir de los cuáles se puede determinar el tamaño proporcional de la operación.

22 Davies, 2018, p. 35.

${ }^{23}$ Idem. El sponsor es un tercero experto, registrado ante la FCA, que revisa y garantiza que la sociedad que patrocina cumpla con la regulación contemplada en las Listing Rules para su inscripción "premium" (premium listing) y para que, durante el tiempo en que se encuentre inscrita, cumpla con la regulación sustantiva y con las exigencias de transparencia e información. Para lo anterior, en varias ocasiones las Listing Rule exigen que la sociedad inscrita consulte a su sponsor (véase Listing Rules, capítulo 8, Sponsors: Premium listing).

${ }^{24} \mathrm{La}$ entonces Superintendencia de Valores y Seguros determinó, mediante Oficio Circular 368 de 16 de octubre de 2006, la adopción obligatoria de las reglas contables IFRS para los emisores de valores en Chile a partir de 2009 . 
eficaces. Por tanto, una política de habitualidad demasiado amplia puede dar lugar a problemas en la regulación de las OPR.

Todo lo anterior deja de manifiesto que el problema de la habitualidad y de las operaciones de bajo monto es un problema común en las jurisdicciones que contemplan regulación de OPR. Pero muestra, también, que existen ciertas mejoras institucionales que pueden ser adoptadas en nuestro sistema jurídico.

\section{Los problemas en el diseño de la política de habitualidad}

Sostiene Enriques que lo esencial en la elaboración de una regulación de OPR es el logro de la mayor eficacia posible, para evitar el riesgo de operaciones de tunneling, pero no de cualquier manera. En sus propias palabras, "el aspecto clave es cómo minimizar aquel riesgo (esto es, tener reglas que son suficientemente efectivas para permitir pocos 'falsos negativos') sin sofocar transacciones que crean valor (esto es, evitar lo más posible los 'falsos positivos') y, de manera más general, sin imponer costos excesivamente altos" $^{25}$. El diseño institucional de la política de habitualidad afecta la amplitud del ámbito de aplicación de las reglas procedimentales para la aprobación a priori de este tipo de operaciones. Por consiguiente, su diseño institucional debe analizarse a la luz del mismo estándar, es decir, verificando que permitan la mayor eficacia posible de la regulación, pero al menor costo, esto es, evitando falsos positivos y disminuyendo los costos para la sociedad en consideración a los beneficios que se pretende lograr ${ }^{26}$.

En Chile, la política de habitualidad debe ser elaborada por el directorio (artículos 147 inciso final letra a LSA y 171 NRSA). A partir del tenor de la disposición reglamentaria, la política de habitualidad se debe incluir en una "autorización de aplicación general”, por medio de esta el directorio determina las condiciones con las que operan las excepciones al procedimiento para aprobación de OPR de inciso final del artículo 147 LSA. No obstante, se observa que es usual que la política de habitualidad se publique independientemente, a partir de lo establecido en el artículo 147 inciso final letra b LSA, que obliga a informar la política de habitualidad poniéndola a disposición de los accionistas en las oficinas sociales o, de haberlo, en el sitio de internet de la sociedad.

La política de habitualidad debe informarse como hecho esencial. En cambio, las operaciones que, siendo entre partes relacionadas, no se sujeten al procedimiento de aprobación por considerarse contempladas en la política de habitualidad por la propia sociedad, solo se deben informar como hecho social "cuando corresponda" (artículo 147

25 EnRIQUes, 2014, p. 14.

${ }^{26}$ Pues en muchas ocasiones las OPR son eficientes. En efecto, se considera que las OPR resultan eficientes cuando los terceros u outsiders no pueden ofrecer condiciones más favorables de negociación a la sociedad que los propios insiders, pues los primeros enfrentarían costos exageradamente altos para hacerse de tal información, o la sociedad debería entregarles secretos comerciales valiosos para ella. Esto explica que las transacciones entre partes relacionadas no sean derechamente prohibidas (en este sentido, ENRIQUES et al., 2017, p. 146 y Kershaw, 2012, p. 478). 
inciso final letra c LSA) o, como indica el reglamento, cuando el directorio lo considere pertinente (artículo 171 NRSA) ${ }^{27}$.

Una vez vigente la política de habitualidad, "no será necesario que el directorio se pronuncie específicamente acerca de cada operación exceptuada” (artículo 171 NRSA), con la salvedad, claro está, de que la propia política de habitualidad así lo exigiera, pues corresponde al propio directorio establecer si se reconocerá o no un mecanismo especial para la aprobación de las operaciones habituales o, al menos, de algunas de ellas determinadas por materia o relevancia a partir de su monto.

El directorio no es el único órgano social que tiene injerencia en la política de habitualidad y en las operaciones celebradas conforme a ella. Por el contrario, la LSA contempla en dos reglas la participación del comité de directores a propósito de OPR.

En primer lugar, el comité de directores puede declarar que una determinada transacción es operación entre partes relacionadas (artículo 146 N 4 LSA), con lo que queda sujeta a la regulación del artículo 147 LSA. Asimismo, puede determinar que una operación entre partes relacionadas exenta de someterse al procedimiento reglado, en virtud de alguna de las letras del inciso final del artículo 147 LSA, quede sujeta de todas formas a su aprobación por tal procedimiento. Con esto, en términos prácticos, el comité de directores puede excluir la aplicación de la política de habitualidad en casos concretos $^{28}$. Es difícil comprender esta norma de otro modo, pues en tal caso la regla sería redundante, ya que el hecho de que la operación se encuentre descrita en la política de habitualidad significa que se trata de una operación entre partes relacionadas. Por lo anterior, la referencia legal a que el comité de directores cuenta con la facultad de declarar que una determinada transacción es operación entre partes relacionadas, "aun cuando se trate de aquellas indicadas en el inciso final del artículo 147”, no puede sino entenderse como que el directorio cuenta con la facultad legal de excluir la aplicación de las exenciones al procedimiento reglado. Esto significa que la ley otorga al comité de directores una forma de evitar falsos negativos cuando sospecha que una OPR puede dar lugar a tunneling, lo que implica que es relevante que el comité tenga la facultad de revisar todas las operaciones.

En segundo lugar, el artículo 50 bis inciso octavo $\mathrm{N}^{\circ} 3$ LSA establece que el comité de directores debe evacuar un informe respecto de los antecedentes relativos a las operaciones del título XVI, sin que tal exigencia legal excluya del informe a las

${ }^{27}$ Esto significa que el directorio deberá analizar si la respectiva operación entre partes relacionadas, aun a pesar de considerarse incluida en las operaciones descritas en la política de habitualidad, cumple sustancialmente con la exigencia de ser una operación que un hombre juicioso consideraría importante conocer para adoptar sus decisiones de inversión (artículo 9 Ley N $\mathrm{N}^{\circ} 18.045$ de 1981 de Mercado de Valores, en adelante LMV). En este sentido, la letra h del punto 4 de la sección acerca de hechos esenciales de la Norma de Carácter General N 30 de 1989, expresa que es hecho esencial: "la suscripción, modificación o término de contratos o convenciones de cualquier especie con personas o entidades relacionadas con la propiedad o gestión de la empresa, cuando involucren montos significativos o revistan relevancia por cualquier causa para la marcha de la empresa, y en la medida que sea distinta de las operaciones habituales mantenidas con las personas o entidades relacionadas".

${ }^{28}$ En contra, Eyzaguirre y Valenzuela, 2015, p. 287. 
operaciones exceptuadas del procedimiento de aprobación según el artículo 147 inciso final. Este artículo podría entonces ser leído como conteniendo una regla que obliga a la sociedad a que las OPR, aun cuando se encontrasen incorporadas en la política de habitualidad, deben ser todas ellas revisadas por el comité de directores e informadas. La cuestión dudosa corresponde al sentido que debe atribuirse a la expresión "examinar los antecedentes", pues podría considerarse que el comité de directores tiene la facultad de aprobar o rechazar la operación ${ }^{29}$. No obstante, tanto la doctrina jurídica que ha abordado esta cuestión como el propio regulador (CMF), en su oportunidad, han entendido que el comité de directores no está obligado a pronunciarse por todas las OPR ${ }^{30}$. En nuestra opinión, lo anterior confirma que el comité de directores puede acceder a toda la información concerniente a operaciones entre partes relacionadas, exceptuadas del procedimiento reglado o no, pues de otra manera no podría emitir un pronunciamiento.

Se observan en la regulación de la política de habitualidad ciertas debilidades que pueden dar lugar a que pueda utilizarse como instrumento para facilitar tunneling. Afirmar lo anterior no debe provocar extrañeza, particularmente luego de que existe evidencia anecdótica al respecto, provista por el caso Cascadas ${ }^{31}$. Primero, la circunstancia de que sea el propio emisor quien determine qué operaciones han de ser consideradas habituales, sin restricciones expresas en la normativa de las sociedades anónimas ${ }^{32}$. Segundo, que no se establezca una restricción cuantitativa a las operaciones declaradas habituales por la propia sociedad. Así lo ha sostenido la CMF, al resolver que las excepciones al procedimiento legal para la aprobación de la operación entre partes relacionadas son

29 Así parecen interpretarlo BUCHuk et al., 2014, p. 2011, al expresar que "las transacciones entre partes relacionadas deben ser aprobadas por un comité presidido por un director independiente".

${ }^{30}$ Eyzaguirre y Valenzuela, 2015, p. 287, haciendo referencia al Oficio circular $\mathrm{N}^{\circ} 560$ de 22 de diciembre de 2009.

${ }^{31}$ Las sociedades Norte Grande y Oro Blanco, con fecha 28 de junio de 2012, modificaron sus políticas generales de habitualidad, especificando con mayor precisión y claridad que en los documentos previos, que la compra y venta entre sociedades pertenecientes a la cascada constituían "operaciones habituales con personas relacionadas dentro del giro ordinario de la Compañía”. Los minoritarios de estas sociedades, particularmente los inversionistas institucionales, reclamaron por este hecho ante la CMF, pues ya se encontraban indagando sobre las operaciones sospechosas por las cuales serían posteriormente sancionados los ejecutivos de las cascadas (Resolución Exenta No 223 de 2 de septiembre de 2014), y vieron en esta ampliación una maniobra para ocultar la información. En un informe en derecho presentado por las AFP inversionistas en las sociedades cascadas, a partir de la disputa legal por la entrega de la información relativa a las operaciones cuestionadas, YrarrázAVAL, 2013, p. 13 sostuvo que mediante la ampliación de la política de habitualidad, los directorios de Norte Grande y Oro Blanco "han evitado poner en conocimiento de los accionistas sus operaciones con partes relacionadas".

${ }^{32}$ No obstante, Eyzaguirre y Valenzuela, 2015, p. 286 consideran que la discreción del directorio en la configuración de la política de habitualidad está restringida: "estimamos que la decisión de adoptar una política de habitualidad debe estar siempre justificada por las actividades propias de la S.A. abierta, referirse a aquellas operaciones realizadas dentro de su giro y que sean recurrentes en su operación, debiendo ser no solo habituales en la práctica de negocios, sino también ordinarias en cuanto a su especie, monto y procedimientos. De la misma manera, esta prerrogativa que entrega la LSA no debe ser ejercida en forma abusiva. Así, por ejemplo, no debiera tolerarse que bajo el amparo de una Autorización General se celebren, como operaciones habituales, transacciones no recurrentes o que sean relevantes con el controlador, ya sea en forma directa o indirecta". 
autónomas. Por lo mismo, una determinada operación, aun siendo de monto relevante, puede quedar excluida por haber sido incorporada a la política de habitualidad ${ }^{33}$. Si bien ese es el diseño legal razonable y esperable y, por tanto, la interpretación de la CMF es correcta, el problema es que se entregue a la propia sociedad la determinación de reglas de aprobación especiales para operaciones habituales que, sin ser técnicamente de monto relevante a partir de la definición legal del artículo 147 letra a LSA, sean transacciones de montos altos. Lo anterior nos sugiere la tercera debilidad: que no se contemplan reglas especiales para la aprobación previa de las operaciones habituales, como, por ejemplo, algún procedimiento simplificado de aprobación ante un órgano ad hoc con representación de minoritarios. Por último, en cuarto lugar, que no se exija que se contemplen mecanismos objetivos para la verificación de que la operación se realiza a precio de mercado, al menos las de montos elevados ${ }^{34}$.

Desde luego, todas estas cuestiones pueden ser resueltas por vía de autorregulación, vale decir, las propias sociedades sujetas a la regulación del título XVI LSA podrían haber contemplado exigencias en este sentido en sus políticas de habitualidad, con el fin de generar mayor confianza en sus inversionistas. Por lo mismo, se hace necesario observar empíricamente si las políticas de habitualidad de, al menos, las sociedades más relevantes del mercado nacional en cuanto a montos de sus acciones transadas, contemplan reglas que prevengan un posible mal uso de la política de habitualidad.

\section{LA OBSERVACIÓN EMPÍRICA DE LAS POLÍTICAS DE HABITUALIDAD EN EL MERCADO CHILENO}

Para verificar el efecto de las políticas de habitualidad en el mercado chileno, observamos las políticas publicadas de las empresas incluidas en el Índice de Precios Selectivo de Acciones de la Bolsa de Comercio de Santiago (en adelante, IPSA) a diciembre de 2017. Si bien a esa fecha este índice incluía a 40 empresas $^{35}$, se obtuvo información para 35 de ellas ${ }^{36}$. La razón para escoger esta muestra estriba en que se trata de las sociedades de mayor transacción bursátil, por lo que cabría esperar que, al ser las más observadas

33 Oficio ordinario $\mathrm{N}^{\circ} 12473$ de 3 de mayo de 2011 de la CMF.

34 Si bien los redactores del Nuevo Reglamento de Sociedades Anónimas (Decreto Supremo de Hacienda $\mathrm{N}^{\circ} 702$ de 2011), en su artículo 172, tuvieron la prudencia de expresar que las OPR exceptuadas del procedimiento de aprobación del artículo 147 LSA, quedan de todas formas sujetas a las exigencias sustanciales de interés social y condiciones de mercado que exige el mismo artículo en su inciso primero, criterio que fue confirmado por la CMF, en la Resolución Exenta No 223 de 2 de septiembre de 2014, p. 677 (caso Cascadas).

35 A partir de septiembre de 2018, el índice IPSA está compuesto por 30 Sociedades.

${ }^{36}$ La CMF, en oficio ordinario N No 9856 de 16 de abril de 2018, nos informó que estas sociedades no habían informado que contaban con política de habitualidad por medio de hecho esencial. De ellas, tres sociedades nos respondieron que no han aprobado una política de habitualidad, en algunos casos justificándolo en el hecho de ser sociedades de inversión, que no realizan operaciones con relacionadas. Por su parte, una sociedad que se incluye en la muestra, SMSAAM S.A., informa la política de habitualidad de su filial, SAAM S.A. 
por los inversionistas, sean las que tengan mayores incentivos para contar con y publicitar procedimientos anti tunelling. Además, debido a que se trata de empresas de mayor tamaño, cuentan con suficiente financiamiento para asesoría legal y de gobiernos corporativos, lo que les permitiría la elaboración de políticas de habitualidad sofisticadas.

Casi la totalidad de las empresas analizadas tienen un controlador ${ }^{37}$. La participación accionaria del controlador es en promedio de 53,9\%, mientras que esos mismos controladores cuentan con, en promedio, derechos sobre el 46,4\% del flujo de caja de esas empresas $^{38}$. En 7 de las 35 empresas consideradas en la muestra de análisis el controlador es de origen extranjero. Los Fondos de Pensiones participan en la propiedad accionaria de 33 de estas empresas, con una participación promedio de 10,4\% en las empresas que participan ${ }^{39}$.

La base de datos incorpora observaciones acerca de aspectos de procedimiento y aspectos de fondo ${ }^{40}$. Los primeros consisten en la verificación de si la sociedad observada contempla mecanismos de control especiales para celebración de OPR. Los segundos, en la calificación expresa de ciertos tipos de operaciones como operaciones habituales.

\section{Aspectos de procedimiento observados en las políticas de habitualidad}

\section{a) Restricciones de monto}

En primer lugar se revisa si existe una restricción del monto de las operaciones que se pueden aprobar sin recurrir al procedimiento reglado, o bien, si se exige que las operaciones no sean de monto relevante. Esto último podría parecer redundante, pues cabría pensar que es una mera repetición del artículo 147 inciso final letra a LSA. Sin embargo, la referencia es relevante, debido al diseño legal, confirmado por la interpretación del regulador, en orden a que cada excepción (letras a, b y c) son aplicables de manera autónoma, con lo que una operación habitual puede ser de monto relevante y, a pesar de ello, quedar exenta del procedimiento reglado para la aprobación de la transacción ${ }^{41}$.

${ }^{37}$ Información a diciembre de 2017. La única empresa perteneciente al índice IPSA que no tiene un controlador (definido según el título XV de la Ley $\mathrm{N}^{\circ}$ 18045) es Nueva La Polar.

${ }^{38} \mathrm{La}$ diferencia entre derechos de control y derechos de flujo de caja es propia de empresas donde exista una cadena de control de tipo piramidal: por ejemplo, la Compañía Sudamericana de Vapores es controlada por el Grupo Luksic por intermedio de Quiñenco $(20,41 \%)$ y sus filiales Inversiones Río Bravo $(33,86 \%)$ e Inmobiliaria Norte Verde (1,90\%), con un total de 56,17\% de las acciones. A su vez, la Familia Luksic controla Quiñenco mediante distintas sociedades, sumando una participación del 81,4\%. Esto implica que la familia Luksic es beneficiaria del 45,7\% (81,4\%*56,17\%) del flujo de Caja de la Compañía Sudamericana de Vapores. La diferencia entre los derechos de control y de flujo de caja en el caso chileno es relativamente baja en comparación a otras economías. En Corea, por ejemplo, tal diferencia alcanza el 40\%. LARRAÍn y URZÚA, 2014, p. 666.

39 En el caso de los Fondos de Pensiones se muestra la participación directa en la Sociedad y no la participación indirecta por medio de estructuras piramidales.

${ }^{40}$ Se puede acceder a la base de datos completa en la siguiente dirección web: https://www.academia. edu/37715899/Base_de_Datos_Articulo_OPR_Lagos-Islas.xlsx

41 Oficio ordinario $\mathrm{N}^{\circ} 12473$ de 3 de mayo de 2011 de la CMF. 
La mención expresa a la exigencia de que la operación no sea de monto relevante en la política de habitualidad, revierte la conclusión anterior, con lo que ese límite aplica a las operaciones aun cuando se encuentren incluidas en la política de habitualidad. Además, se verifica si existe una regla de agregación de montos, como la de la letra a del inciso final del artículo 147 LSA, o una más exigente, pues se trata de una regla antielusión que hace más efectivo el control ${ }^{42}$.

Solo en 5 de las 35 empresas, la política de habitualidad establece límites máximos para que una operación sea considerada como habitual. En 2 de estas 5 empresas la política de habitualidad simplemente repite los límites establecidos en la ley, por lo que solo en 3 casos (8,5\% del total) las Sociedades Anónimas establecen un límite para la habitualidad por debajo del monto definido por la ley ${ }^{43}$. En las otras 30 empresas no existe límite, lo que, debido a la interpretación que realizó la CMF, implica que, potencialmente, operaciones de montos significativos pueden quedar dentro de la categoría de habituales.

\section{b) Procedimiento para aprobación de operaciones con filiales o intragrupo}

En segundo lugar, se verifica si se contempla alguna restricción o mecanismo especial para operaciones con filiales, como por ejemplo la exención de control de la letra c del inciso final del artículo 147 LSA, que exige una participación de 95\%, o bien, un nivel de concentración menor. Asimismo, se revisa si se establecen reglas especiales acerca de operaciones con otras empresas del grupo, pues en un sistema de propiedad concentrada como el chileno suponen un mayor riesgo de expropiación a inversionistas ${ }^{44}$. En el caso de las 35 empresas analizadas, para el caso de las empresas filiales, en 4 empresas se observa que la política de habitualidad relaja la restricción del 95\% de participación en empresas filiales. Por otra parte, en el caso de la relación con otras empresas del controlador, solo 2 empresas establecen normas especiales: una de ellas lo hace en la dirección de facilitar las transacciones con el controlador y sus empresas, mientras que, en el segundo caso, se establecen restricciones que prohíben los préstamos de la empresa y sus filiales al accionista controlador ${ }^{45}$.

\footnotetext{
${ }^{42}$ En el mismo sentido, la Listing Rule 11.1.11 impone la regla de agregación de 12 meses.

43 Este porcentaje es similar a lo que encuentran BIANCHI et al., 2014, p. 32. en su análisis de los Códigos de Autorregulación de Empresas Italianas.

${ }^{44}$ Por ejemplo, en Italia se reduce la ratio utilizada para considerar si una operación es de monto relevante, de $5 \%$ a 2,5\% cuando se trata de operaciones con la matriz (BIANCHI et al., 2014, p. 11). Por su parte, DAviEs, 2018, p. 36, da a entender que sería sensato distinguir entre montos relevantes para sociedades relacionadas y montos relevantes para personas naturales relacionadas, pues lo que para una sociedad puede ser poco, para una persona puede ser mucho.

4514 de las 35 empresas analizadas son parte de una estructura de propiedad de tipo piramidal, las que han sido identificadas en la literatura como aquellas en donde pueden existir mayores incentivos para conductas tipo tunneling por parte de los controladores.
} 


\section{Aspectos de fondo observados en las políticas de habitualidad}

En lo que respecta a las actividades que cada empresa incluye o considera como habituales, podemos distinguir entre aquellas que son propias del giro de cada empresa $y$, por ende, es posible presumir que la transacción con una relacionada responde a aspectos de eficiencia operativa ${ }^{46}$, versus aquellas que se alejan del giro, y, por tanto, pueden ser materias particularmente riesgosas en términos de tunneling. A continuación se analizan cuatro de esas materias. La inclusión de estas materias se funda en que se trata de aquellas que tanto la literatura teórica como la experiencia práctica (en Chile y en el exterior) reconocen como aquellas donde existe un mayor riesgo de extracción indebida.

\section{a) Asesorías y estudios}

En primer lugar, "asesorías" y estudios financieros o de otras materias, como administración o marketing, pues se trata de servicios cuyo precio de mercado es muy difícil de evaluar y cuya prestación efectiva es compleja de acreditar. Esto determina un aumento del riesgo de que estas operaciones sean utilizadas como mecanismo de extracción de valor.

En 26 de las 35 empresas $(74,28 \%)$ las asesorías y estudios se consideran como operaciones habituales. Al analizar según las características de cada empresa, no se encuentran diferencias significativas ya sea en relación con el tipo de controlador (nacional o extranjero) o al porcentaje de participación en el control de la empresa.

\section{b) Cuenta corriente}

En segundo lugar, la cuenta corriente mercantil, pues existe una comprensión jurídicamente errónea de la institución muy extendida en la práctica nacional, conforme a la cual la cuenta corriente es causa suficiente para justificar un negocio jurídico registrado en la cuenta y no, como realmente lo es, un contrato accesorio para desarrollar una relación de negocios de largo aliento mediante compensaciones y eventuales otorgamientos de crédito para el pago de saldos ${ }^{47}$. En 21 de 35 Sociedades, la cuenta corriente mercantil

\footnotetext{
${ }^{46}$ Por ejemplo, la política de habitualidad de Concha y Toro, incluye dentro de las operaciones habituales "la compra y venta de fruta, uvas y vinos productos similares, hasta la cantidad de UF10.000; la prestación de servicios de promoción y publicidad de vinos y la intermediación y comisión de vinos en el extranjero". Mientras que en el caso de Colbún, se consideran como habituales "la compra y venta de energía eléctrica, tanto en el mercado regulado como a clientes libres, ya sea bajo contratos de suministro o no”.

${ }^{47}$ En este sentido Pérez de la Cruz, 2011, p. 113. En el mismo sentido, De Eizaguirre, 1994, p. 348, al señalar: "la relación negocial constituye el antecedente o soporte del contrato de cuenta corriente, en cuanto que en aquella tienen su origen los créditos y pagos que sirven de alimento a la cuenta corriente. Dicha relación puede consistir en una vinculación duradera, como es el caso de las relaciones bancarias de apertura de crédito o de depósito de numerario, o la de factoring, o en una serie de relaciones repetidas de tracto único, como las propias del contrato de comisión, en sus modalidades de venta o de transporte. La relación tiene su origen en un contrato, que es el que nos indicará la existencia, en su caso, y dentro de qué límites, de la concesión de crédito”. El caso de un préstamo entre matriz y filial que dio lugar a la sanción
} 
se incluye dentro de las operaciones habituales. Es importante notar que esta inclusión es más frecuente en el caso de las empresas cuyo controlador es extranjero versus aquellas cuyo controlador es nacional.

\section{c) Contratación de créditos}

En tercer lugar, la contratación de crédito, pues es un tipo de operación que característicamente la literatura económica de gobiernos corporativos revisa para verificar si existe tunneling ${ }^{48}$.

Para el caso de las contrataciones de crédito, se restringe el análisis para una submuestra que considera 29 empresas no financieras (ya que para el caso de las empresas financieras, la contratación de créditos es parte del giro habitual de los negocios). En 24 de estas empresas $(82,7 \%$ ) la contratación de créditos queda incluida dentro de las operaciones habituales. Al igual que en los casos de las asesorías, no hay diferencias significativas entre empresas cuyo controlador sea local o extranjero.

\section{d) Adquisición de títulos de sociedades relacionadas}

En cuarto lugar, la adquisición de acciones o títulos de relacionadas. En este caso, existe potencial de tunneling toda vez que el controlador puede tener porcentajes distintos de participación en distintas sociedades, lo que abre una oportunidad para extraer valor de los accionistas minoritarios por la vía de las transacciones de acciones, especialmente en aquellos casos donde existan estructuras de tipo piramidal. Es admisible notar a que este fue el tipo de operación por medio del cual se realizó tunneling en el caso Cascadas ${ }^{49}$, y que, por otra parte, la evidencia empírica para el caso chileno muestra que los controladores pueden extraer valor por parte de los inversionistas controlando el timing de emisión de nuevas acciones ${ }^{50}$.

En comparación con los aspectos revisados anteriormente, la adquisición de acciones aparece en forma menos frecuente en las políticas de habitualidad de las sociedades integrantes del IPSA: solo en 12 de las 35 empresas se consideran como operaciones habituales $^{51}$. En este ítem, existe una diferencia relevante entre las sociedades cuyo controlador es extranjero versus aquellas con un controlador local. Mientras que, en las primeras, solo 1 de 8 sociedades $(12,5 \%)$ incluye las transacciones de acciones, en el

\footnotetext{
impuesta a los directores de Ohio National Seguros de Vida S. A. por la CMF ilustra la aludida comprensión jurídicamente errónea de la institución (Resolución exenta 4176 de 11 de octubre de 2016).

${ }^{48}$ Véase para Chile Buchuk et al., 2014, pp. 190-212.

${ }^{49}$ Resolución Exenta No 223 de 2 de septiembre de 2014.

${ }^{50}$ Véase Larraín y URzúa, 2013, pp. 661-681.

${ }^{51}$ En 9 sociedades la autorización es explícita, mientras que en otras 3 la autorización es implícita, ya que las políticas de habitualidad incluyen cláusulas suficientemente amplias como para incluir las transacciones de acciones. Por ejemplo, la Política de Habitualidad de Parque Arauco en su inciso 1) incluye "La adquisición, desarrollo, explotación, venta, enajenación y negociación en cualquier forma y a cualquier título, de toda clase de bienes muebles o inmuebles, corporales e incorporales, ya sea en Chile o en el extranjero".
} 
caso de las sociedades cuyo controlador es local, 11 de 27 sociedades (40,7\%) incluye la adquisición de acciones como parte de sus operaciones habituales.

\section{Tabla resumen de resultados de la observación}

La siguiente tabla resume los principales resultados. En la tabla se puede apreciar cómo, por una parte, las sociedades actúan de modo pasivo, pues la gran mayoría de las políticas de habitualidad de las sociedades anónimas observadas no profundizan en los procedimientos ni establecen restricciones adicionales a lo señalado en la ley. Pero, por otra parte, son activas al adoptar, en la mayoría de los casos observados, definiciones amplias de lo que se considera como una operación habitual.

Tabla resumen de resultados: políticas en relación a operaciones con partes relacionadas

\begin{tabular}{|c|c|c|}
\hline & Sí & No \\
\hline \multicolumn{3}{|c|}{$\begin{array}{l}\text { La política de habitualidad incorpora restricciones y procedimientos adicionales } \\
\text { a lo señalado en la ley }\end{array}$} \\
\hline $\begin{array}{l}\text { La política establece restricciones de montos a las operaciones que son } \\
\text { consideradas habituales }\end{array}$ & 5 & 30 \\
\hline Restricciones adicionales a operaciones relacionadas con el controlador & 1 & 34 \\
\hline \multicolumn{3}{|c|}{ La política incluye dentro de su definición de habitualidad a: } \\
\hline Operaciones de cuenta corriente mercantil & 21 & 14 \\
\hline Asesorías o estudios financieros o de otras materias & 26 & 9 \\
\hline Contratación de créditos $(*)$ & 24 & 5 \\
\hline Adquisición de acciones $(*)$ & 12 & 23 \\
\hline
\end{tabular}

Al agregar los 4 ítems correspondientes a la definición de actividades consideradas como habituales, es posible construir un índice de habitualidad, el que toma valores que van desde 4 (para el caso de las empresas donde los 4 tipos de operaciones analizadas anteriormente son consideradas como habituales) a 0 (si la política de habitualidad no contempla ninguno de los ítems analizados). Esto implica que aquellas sociedades donde el índice toma un valor mayor, el potencial de tunneling es también mayor.

El índice promedio para las 29 empresas no financieras alcanza un valor de 2,62, lo que da cuenta de que la mayoría de las empresas elige un criterio amplio para clasificar una transacción como habitual. No existe una relación estadísticamente significativa entre el valor del índice y la participación accionaria del mayoritario, así como se aprecia en la siguiente figura 
Índice de habitualidad y participación del controlador

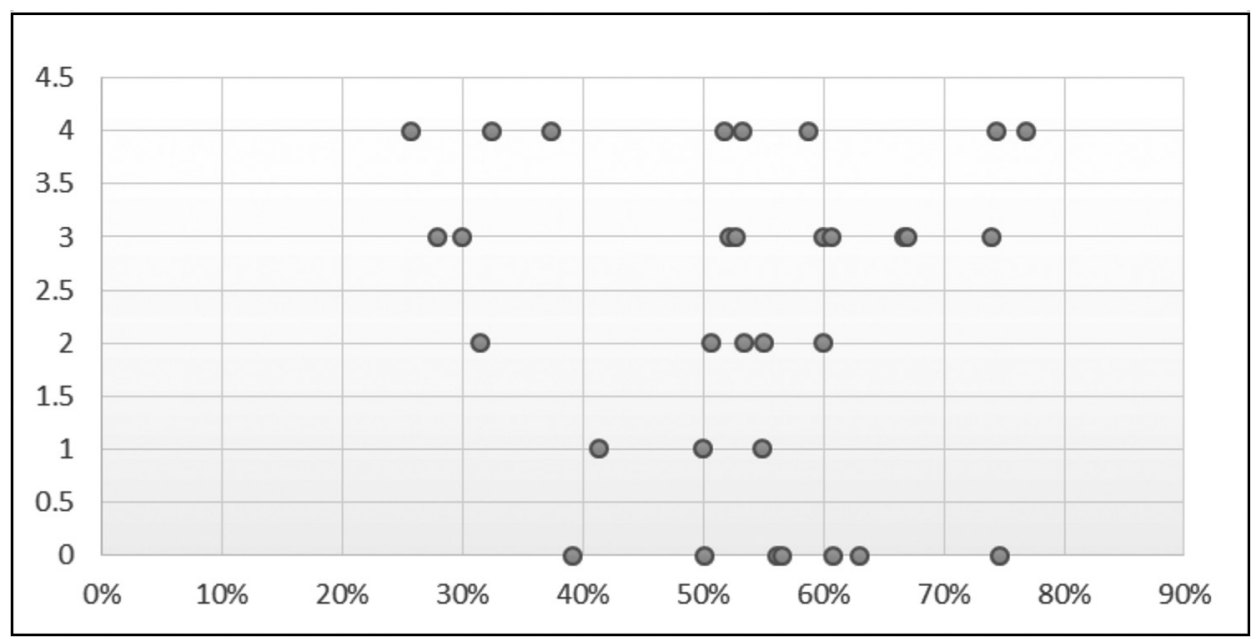

\section{CONCLUSIONES Y PROPUESTAS}

El análisis empírico de las políticas de habitualidad de las sociedades anónimas del IPSA indica que existe poco incentivo a la autorregulación. A partir de esta constatación y del análisis de la experiencia comparada y la doctrina realizado en este trabajo es posible proponer lo siguiente:

1. De las empresas observadas, la mayoría no cuenta con límites de monto para la calificación de una transacción como habitual, lo que si se combina con la autonomía de las excepciones al procedimiento reglado, permite que en la mayoría de los casos puedan considerarse habituales transacciones de cualquier monto, aun si se trata de sumas elevadas. Esta regla legal, reafirmada por una interpretación administrativa, debe modificarse incorporando matices, esto es, estableciendo mecanismos de control simplificados. En su estado actual, la regla genera un evidente riesgo de tunneling, como demostró el caso Cascadas ${ }^{52}$. Es razonable que las sociedades, o el propio regulador si en el futuro la ley le reconoce esa facultad, incorporen sublímites, para que se puedan establecer exigencias mínimas de procedimiento por tramo, como aprobación del comité de directores, licitación u otras, liberando de todo control solo a las de monto realmente reducido. En el mismo

52 Véase Boletín No 10.162-05 indicaciones 17-07-2017 que, entre otras materias, modifica las reglas respecto de política de habitualidad. En lo pertinente a este punto, establece un baremo de $10 \%$ del activo social, límite que es aún demasiado alto, pues la reforma propuesta no sugiere procedimientos simplificados para la aprobación de estas operaciones. 
sentido, las operaciones entre empresas de un mismo grupo empresarial, que son las más riesgosas, deberían contemplar un mecanismo especial de aprobación y mayores exigencias de información, aun cuando no queden sujetas al procedimiento reglado en virtud de su incorporación a la política de habitualidad.

2. El comité de directores debería jugar un rol relevante tanto para la elaboración ${ }^{53} y$ aprobación de la política general de habitualidad como para la revisión de ciertas operaciones habituales, particularmente aquellas que se incorporen a los tramos de montos superiores. No obstante, para que este órgano juegue un rol efectivo es indispensable que los directores disidentes o externos (no elegidos por el controlador) constituyan mayoría en el comité, cuestión que no siempre ocurre con los niveles de concentración de propiedad que se observan en el mercado chileno ${ }^{54}$.

3. A pesar de que se encuentra que las políticas de habitualidad son poco sofisticadas y tienden a repetir la ley, las sociedades en su mayoría $(74,28 \%)$ tienden a incluir, dentro de las operaciones habituales, a las "asesorías". Esto da a entender que se trata de una práctica extendida. Obviamente, es difícil sugerir mecanismos objetivos de valoración, pero sí es posible proponer mayores exigencias de información en este tipo de operaciones, declarando específicamente el sentido y utilidad de cada asesoría realizada. Sería esencial tener presente que en estos casos no se está entregando información relativa a precios de operaciones con proveedores, que pueden ser útiles para competidores y, por tanto, eventualmente perjudiciales de divulgar para la respectiva sociedad. En consecuencia, mayores exigencias de divulgación no son problemáticas. Asimismo, en lo que respecta a la cuenta corriente, es necesario entregar mayor información respecto de las operaciones que se integran en una relación de cuenta corriente, determinando sublímites de monto y dejando constancia del tipo de operación (por ejemplo, compraventa, mutuo, o suministro) que se integra en la respectiva cuenta corriente ${ }^{55}$. El problema de este tipo de operaciones, como se ha visto, es que su avaluación es compleja. Sería conveniente verificar empíricamente, en futuras investigaciones, si se realiza tunneling por medio de este tipo de operaciones. En cambio, los mutuos entre sociedades son verificables por medio de la información contable que se incorpora en las memorias de las sociedades y, al parecer, esa transparencia da lugar a que no se utilice como mecanismo de tunneling 56 .

\footnotetext{
${ }^{53}$ En este sentido, Boletín No 10.162-05 indicaciones 17-07-2017.

${ }^{54}$ Véase LEFORT y URZúA, 2008, pp. 617-618.

55 Sugerencia para el desglose de información que ha de entregarse a CMF según nuevo inciso final que incorporaría el proyecto de ley que modifica las reglas sobre habitualidad (Boletín N ${ }^{\circ}$ 10.162-05 indicaciones 17-07-2017).

${ }^{56}$ En este sentido, Buchuk et al., 2014, pp. 208-211. No obstante, Urzúa, 2009; Larraín y Urzúa, 2013, p. 662, y Torres et al., 2017, p. 102, encuentran evidencia directa e indirecta de tunneling en el mercado chileno.
} 
La evidencia empírica para Chile demuestra que existe tunneling, y que en ocasiones este tiene lugar por medio de OPR. Pero, al mismo tiempo, no da cuenta que las OPR son utilizadas como mecanismos para reducir los costos de transacción y, por ende, aumentan el valor de las empresas. Vistos en su conjunto, estos estudios destacan la importancia del diseño de políticas que permitan limitar los efectos expropiatorios que pueden tener las OPR pero sin recurrir a prohibiciones que pueden tener impacto negativo en su desempeño.

Este trabajo demuestra que la regulación de la política de habitualidad, en su estado actual, o, más bien dicho, la ausencia de regulación, puede explicar la limitada eficacia de la regulación general de operaciones entre partes relacionadas.

\section{BIBLIOGRAFÍA}

Atria, Fernando, 2015: "Los deberes de los directores de sociedades anónimas frente al régimen general de responsabilidad civil” (inédito).

Barros, Enrique, 2006: Tratado de responsabilidad extracontractual, Santiago, Editorial Jurídica de Chile.

Bianchi, Marcello; Ciavarella, Angela; Enriques, Luca; Novembre, Valerio; Signoretti, Rosella, 2014: "Regulation and self-regulation of related party transactions in Italy. An empirical analysis" European Corporate Governance Institute Working Paper, № 415.

Bona-Sánchez, Carolina; Fernández-Senra, Carmen y Pérez-Alemán, Jerónimo, 2017: "Related-party transactions, dominant owners and firm value", Business Research Quarterly, Vol. 20, $\mathrm{N}^{\circ} 1$.

Buchuk, David; Larrain, Borja; Muñoz, Francisco y Urzúa, Francisco, 2014: "The internal capital markets of business groups: Evidence from intra-grouploans", Journal of Financial Economics, Vol. 112, $\mathrm{N}^{\circ} 2$.

Centro de Gobierno Corporativo UC, 2015: Comentarios proyecto normativo que propone modificaciones a NCG 341 y NCG 30: Fortalecimiento de estándares de gobierno corporativo de las sociedades anónimas abiertas. Recuperado el 17.04.2018: http:// centrogobiernocorporativo.uc.cl/Ver-documento/247-Comentarios-Proyecto-Normativoque-Propone-Modificaciones-a-la-NCG-341-y-NCG-30.html.

DAviEs, Paul, 2014: "Related party transactions: UK model", European Corporate Governance Institute Law Working Paper, $\mathrm{N}^{\circ} 387$.

Deakin, S.; Gindis, D.; Hodgson, G.; Huang, K.; Pistor, K., 2017: "Legal institutionalism: capitalism and the constitutive role of law", Journal of Comparative Economics, Vol. 45, $\mathrm{N}^{\mathrm{o}} 1$.

De Eizaguirre, José María, 1994: "De nuevo sobre el contrato de cuenta corriente", Revista de Derecho Bancario y Bursátil, No 54.

ENRIQUES, Luca, 2014: "Related party transactions: policy options and real-world challenges (with a critique of the european commission proposal)", European Corporate Governance Institute Working Paper Series in Law, No 267.

Eyzaguirre, Cristián y VAlenzuela, Ignacio, 2015: "Las operaciones con partes relacionadas en las sociedades anónimas abiertas”, Actualidad Jurídica, $\mathrm{N}^{\circ} 31$.

Fiss, Owen M., 1983-1984: “Against settlement”, Yale Law Journal, Vol. 93, Nº 6.

Germain, Michel y Magnier, Véronique, 2009: Traité de droit commercial G. Ripert et R. Roblot (19 edición), tome 1 , volumen 2, Paris, L.G.D.J. 
Johnson, Simon; La Porta, Rafael; López de Silanes, Florencio; Shleifer, Andrei, 2000: "Tunneling", The American Economic Review, vol. 90 N 2.

Kershaw, David, 2012: Company law in context ( $2^{\mathrm{a}}$ edición), Oxford, Oxford University Press.

LARraín, Borja y UrzúA, Francisco, 2013: "Controlling shareholders and markettiming in share issuance", Journal of Financial Economics, Vol. $109 \mathrm{~N}^{\circ} 3$.

Lefort, Fernando y Urzúa, Francisco, 2008: "Board Independence, firm performance and ownership concentration: evidence from Chile”, Journal of Business Research, Vol. 61 No 6.

Lefort, Fernando y Wigodski, Teodoro, 2007: Una mirada al gobierno corporativo en Chile, Santiago, Ediciones Universidad Católica de Chile

OECD, 2012: Related party transactions and minority shareholders rights, Recuperado el 17.04.2018. https://www.oecd.org/daf/ca/50089215.pdf

PAZ-Ares, Cándido, 2004: "El gobierno corporativo como estrategia de creación de valor", Revista de Derecho Mercantil, No 251.

Pérez de la Cruz, Antonio: "El contrato de cuenta corriente", en Lecciones de Derecho Mercantil ( $9^{a}$ edic., dirs. A. Menéndez y A. Rojo, A.), Cizur Menor, Civitas-Thomson Reuters.

Proxinvest, 2014: A (not so) useful French Bill on related party transactions. Recuperado el 17.04.2018. http://www.proxinvest.fr/?p=1199\&lang =en

Ross SchneIder, Ben, 2013: Hierarchical capitalism in Latin America, Cambridge, Cambridge University Press.

Schmidt, Dominique, 2009: Des "conventions réglementées" à la publication des transactions entre parties liées. Recuperado el 17.04.2018. https://www.cabinet-schmidt.avocat.fr/doc/ CONVENTIONS_REGLEMENTEES_FINAL.pdf

CMF, 2015: Fortalecimiento de estándares de gobierno corporativo. Informe final. Recuperado el 17.04.2018: https://www.CMF.cl/portal/principal/605/articles-20924_doc_pdf.pdf.

Torres, Juan Pablo; Jara, Mauricio y López-Iturriaga, Félix, 2017: "Corporate Control and Firm Value: The Bright Side of Business Groups”, Journal of Family Business Strategy, Vol. 8, $\mathrm{N}^{\circ} 2$.

UrzúA, F., 2009: "Too few dividends? Groups tunneling through: evidence from intra-group loans", Journal of Corporate Finance, Vol. 15 № 2.

Yrarrázaval Covarrubias, Arturo, 2013: Informe en derecho. Disponible en http://s2.pulso.cl/ wp-content/uploads/2013/09/1816132.pdf

Normas jurídicas citadas

LEY $\mathrm{N}^{\circ}$ 20.382, Introduce perfeccionamientos a la normativa que regula los gobiernos corporativos de las empresas, publicada el 20 de octubre de 2009.

LEY No 18.046, de Sociedades Anónimas, publicada el 22 de octubre de 1981. 